\title{
HYDROSTATIC LEVEL SYSTEM FOR SLOW GROUND MOTION STUDIES AT FERMILAB AND SLAC $*$
}

\author{
A. Seryi, R. Ruland, SLAC, Stanford, CA, 94309 USA \\ B. Baklakov, A. Chupyra, A. Erokhin, M. Kondaurov, A. Medvedko, \\ V. Parkhomchuk, Sh. Singatulin, E. Shubin, BINP, Novosibirsk, 630090 Russia \\ J. Lach, D. Plant, V. Shiltsev, FNAL, Batavia, IL, 60510, USA
}

\section{Abstract}

A series of new ground motion studies using an upgraded hydrostatic level system are planned at Fermilab and SLAC in collaboration with BINP. To better characterize both the spatial and temporal characteristics of slow ground motion, these studies will use large number of probes and will also be performed in several geologically different locations. The hydrostatic level system used in ongoing measurements near Fermilab [1] suffers from uncertainty of temperature fluctuation effects. This paper presents improvements to be incorporated into the upgraded hydrostatic level system, present status, and the plan for experimental studies.

\section{INTRODUCTION}

The electron-positron linear colliders envisioned for the future must focus the beams to nanometer size in order to achieve design luminosity. Small beam sizes impose strict tolerances on the positional stability of the collider components, but ground motion will continuously change the component positions.

For linear colliders, it is useful to separate ground motion into two categories, fast and slow. Fast ground motion (roughly $\mathrm{f}>0.1 \mathrm{~Hz}$ ) causes the beam position to change from pulse to pulse. In contrast, slow ground motion ( $f<0.1 \mathrm{~Hz}$ ) does not result in an offset of the beams at the interaction point since it is corrected by feedback on a pulse to pulse basis. However, in spite of being very slow, this motion can have a rather short wavelength, causing misalignments of the collider, which produce emittance growth as the beam trajectory deviates from the ideal line.

Two distinct types of slow motion have been identified - diffusive [2,3] and systematic [4]. Diffusive motion is dominant over a time scale of hours to days, systematic over months to years. Diffusive motion is a greater concern for a linear collider. It is usually described by the ATL law [2,3] for the RMS relative misalignment of elements: $\Delta \mathrm{X}^{2}=$ ATL where $\mathrm{T}$ is the time since perfect alignment and $\mathrm{L}$ is the distance between points.

The parameter A appears to vary by a few orders of

*Work supported in part by the U.S. Department of Energy, Contract DE-AC03-76SF00515 magnitude when measured in different places and is very site and geology dependent. For example, the DESY HERA slow beam motion were found to be consistent with the ATL law with $\mathrm{A}=10^{-5} \mu \mathrm{m}^{2} / \mathrm{m} / \mathrm{s}$ [5]. Sazaare mine (Japan) measurements [6] report granite tunnel motion with $\mathrm{A}=2 * 10^{-9} \mu \mathrm{m}^{2} / \mathrm{m} / \mathrm{s}$ [6]. For diffusive motion with a value of $\mathrm{A}=5^{*} 10^{-7} \mu \mathrm{m}^{2} / \mathrm{m} / \mathrm{s}$ (similar to observations at SLAC in the FFTB and SLC tunnels $[7,8]$ ) the NLC (Next Linear Collider) linac would require periodic undisruptive (for luminosity) beam based alignment every few hours, assuming that feedback is used to maintain the trajectory at several locations [9].

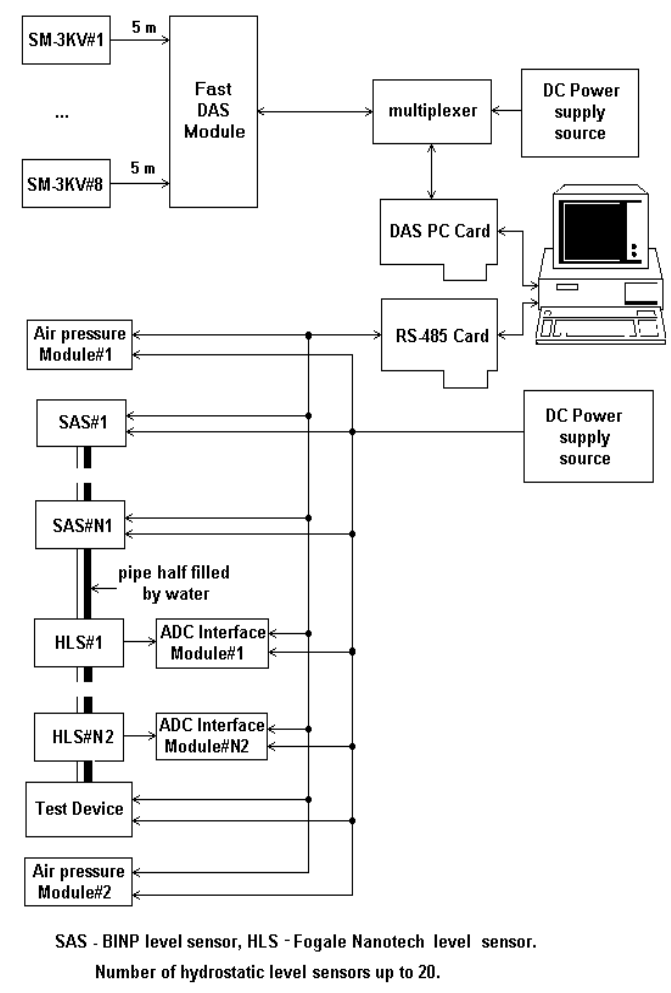

Figure 1: Block diagram of the Slow Ground Motion Measurement (SGMM) System.

The Slow Ground Motion Measurement System (SGMM System) has been developed and is being manufactured at BINP (Budker Institute of Nuclear Physics, Novosibirsk, Russia). This high-resolution 
system will be used to make measurements of slow ground motion in three different geological sites, the Fermilab Main Injector tunnel, the Aurora mine in the Illinois dolimite, and the SLAC PEP-II tunnel. Analysis of the experimental results will be used to refine the site criteria for the NLC project.

\section{SLOW GROUND MOTION MEASUREMENT SYSTEM}

The SGMM System is an universal and powerful system based on an IBM PC. It can simultaneously collect data from up to 20 hydrostatic level sensors of different types, 2 air pressure sensors and up to $8 \mathrm{SM}-3 \mathrm{KV}$ geophones (or similar devices). Measurement results to be presented on a computer display in an operator-friendly manner and stored for later analysis. The block diagram of the SGMM System is shown in Fig.1.

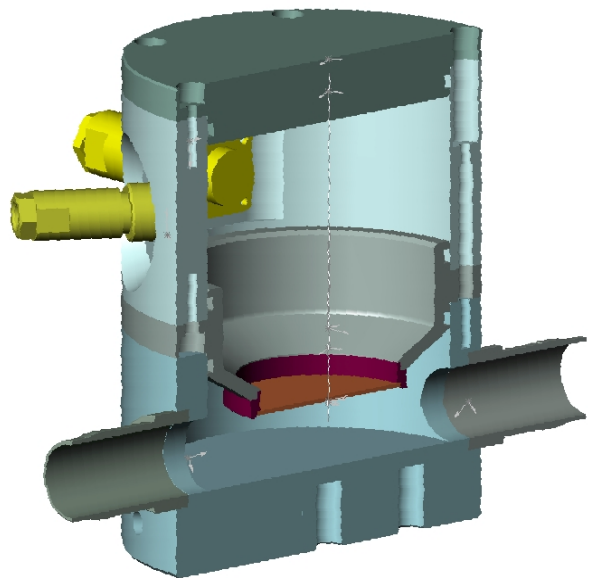

Figure 2: Schematic of the SAS hydrostatic level probe.

The SGMM System includes the Hydrostatic Level Measurement System (HLMS), the Seismic Measurement System (SMS), an operator console and various DC power supplies. The HLMS includes up to 20 hydrostatic level sensors of two types (SAS - BINP sensors shown in Fig. 2 and HLS - Fogale-Nanotech sensors made in France), two air pressure modules and a Hydrostatic System Test Device (TD). The SAS sensors have a builtin RS-485 interface. The Fogale Nanotech sensors (HLS) can be integrated into the system with special RS-485 compatible ADC interface modules (one module per sensor). All sensors are linked by stainless steel tubes that form a circuit of communicating water vessels. The tubes will be with water in half and that will make the system less sensitive to temperature variations compared to a completely filled pipe system, as in [1]. With half-full tubes, the stable water surface is defined only by the constant gravity line. This method for reducing temperature sensitivity in a hydrostatic level system has been used since at least 1914 [10]. It has been adopted for recent ground motion studies in Japan [6]. The SMS consists of a set of seismometers and a fast Data Acquisition System (DAS) module.

The operator console is an IBM PC with an RS-485 interface card and a Data Acquisition System card inside the PC box. The RS-485 interface is a commercially available standard PC card controlling data transfer to and from the remote BINP hydrostatic level sensors, ADC interface modules, air pressure modules and the Test Device. The DAS card is a specially designed board controlling data transfer to/from the remote fast DAS module.

The total length of the RS-485 local area network can be up to $1200 \mathrm{~m}$. The distance between the DAS module and the operator console depends on the quality of the RF cable connecting them and can be as large as $1200 \mathrm{~m}$. The HLMS power to be supplied by a separate cable. The SMS is powered from the same cable that is used for data transmission. All together, the BINP hydrostatic level sensors, ADC interfaces, air pressure modules, Test Device and DAS Module need a standard +48 V 2A DC power supply.

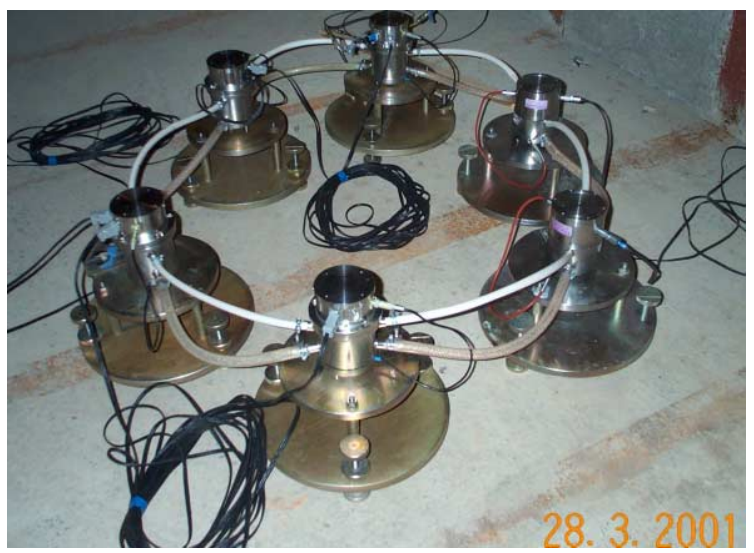

Figure 3: Four SAS and two Fogale probes in the dual tube configuration placed close together to measure system noise.

This system will allow continuous acquisition of slow ground motion measurement data. Data sampling frequencies are up to $1 \mathrm{~Hz}$ for the HLMS hydrostatic level system and up to $1 \mathrm{kHz}$ for the fast seismic measurements with the SMS.

The Test Device (TD) was developed as an integral part of the system to provide online calibration. The TD shifts average water level in the hydraulic system to a preset value by moving an object that is partly submerged in the water.

\section{SGMM TEST}

Several SAS sensors have been manufactured and tested in September - October 2000. For the tests, the probes were connected with dual pipes (for water and for air) and were installed over a distance of about 100 meters in the VEPP5 tunnel at BINP (Novosibirsk). Results are 
presented in Fig.4 where, in particular, the effect of tidal motion is clearly seen.

In the beginning of 2001, an improved version of the SAS sensors was developed with more stable electronics. To use these sensors with half-full tubes, the bottom part of the probe had to be replaced. The new sensors were tested, first with dual tubes (see Fig.3), and then with a single half filled tube. The system noise was determined by placing the probes very close together.

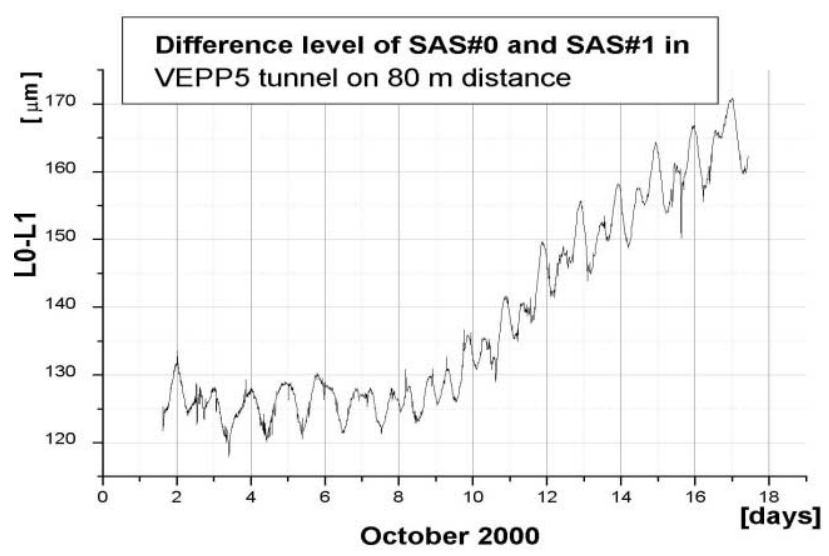

Figure 4: Difference of the levels over $80 \mathrm{~m}$ with two SAS hydrostatic level probe.

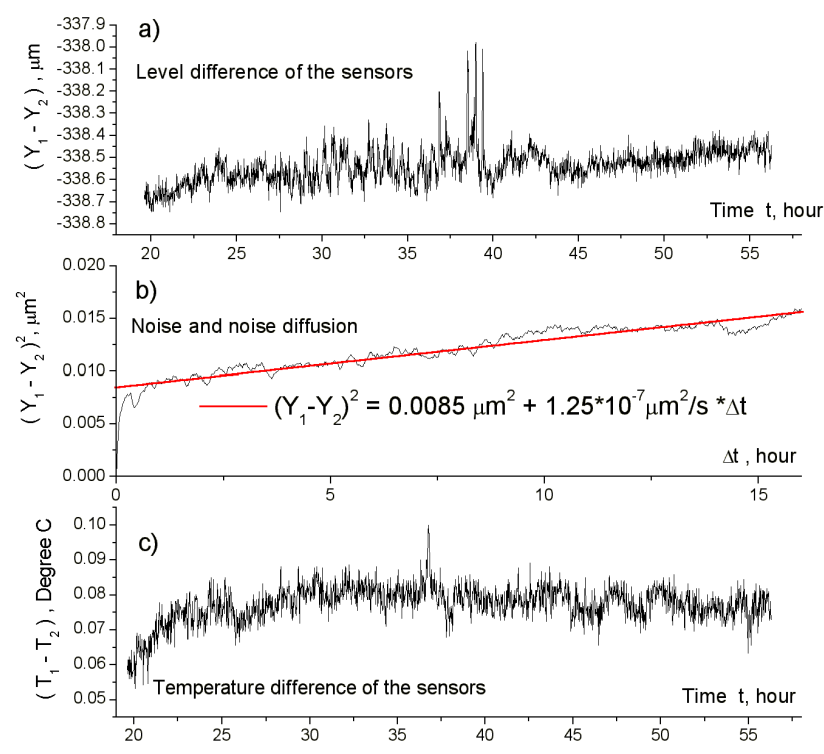

Figure 5: Level difference (a), diffusion (b) and temperature difference (c) of two SAS probes of $2^{\text {nd }}$ iteration of design installed $1 \mathrm{~m}$ apart (April 2001).

These measurements indicate that the system noise consists of two terms - one independent of time, and another proportional to time (see Fig.5): $\sigma^{2}=(0.09 \mu \mathrm{m})^{2}$ $+1.25 \mathrm{E}-7 \mu \mathrm{m}^{2} / \mathrm{s}^{*} \mathrm{~T}$. For $\mathrm{T}=1$ hour time interval, this noise power is factor 4 less than the HLS system noise reported in [1] , and some 40 times less for $\mathrm{T}=2$ days.

Assuming that the system noise behaves similarly when the probes are installed in the tunnel, one can estimate the expected resolution of the system. For a system with a total length of $500 \mathrm{~m}$, the ultimate resolution in the measurement of the ATL coefficient can be about $3 * 10^{-10}$ $\mu \mathrm{m}^{2} / \mathrm{m} / \mathrm{s}$. This resolution would only be achievable for measurements taken over a period longer than $\sim 20$ hours. For shorter periods, the constant term in the noise formula will dominate. For example, for one hour interval measurement, the resolution is about $5^{*} 10^{-9} \mu \mathrm{m}^{2} / \mathrm{m} / \mathrm{s}$. With a shorter system length, such as $50 \mathrm{~m}$ (similar to the betatron wavelength in NLC), the resolution might be about $1.6 * 10^{-8} \mu \mathrm{m}^{2} / \mathrm{m} / \mathrm{s}$. For comparison, the minimum ground diffusion coefficient measured in $250-\mathrm{ft}$ deep Aurora mine in the Illinois dolomite is about $2 * 10^{-7}$ $\mu \mathrm{m}^{2} / \mathrm{m} / \mathrm{s}$ [1]. Therefore, we believe that the new system will allow substantially increase signal to noise ratio in ground motion measurements, compared to [1].

After the system test in April 2001, several additional modifications were made to further improve the performance. In the fall 2001 we plan to install system with 20 probes in one of the beam lines of the Fermilab Main Injector. Neighbor probes will be distanced by about $10 \mathrm{~m}$ - equal to the distance between quadrupole magnets in the NLC project [11]. The existing system in the Aurora mine will be modified to include 4 to 8 new probes. Similar measurements are planned at SLAC.

\section{CONCLUSION}

New hydrostatic level system has been developed for slow ground motion studies at Fermilab and SLAC. New series of measurements will start in fall 2001 with major goal to obtain comprehensive experimental data on both the spatial and temporal properties of slow ground motion. Compared to previous measurements [1], many more probes with much better resolution will be employed. The studies will be performed in several geologically different locations in an effort to refine the site criteria for the Next Linear Collider [11,12].

\section{REFERENCES}

[1] V. Shiltsev, et al, in these Proceedings.

[2] B. Baklakov, et al. Tech. Phys. 38, 894 (1993).

[3] V. Shiltsev, in Proc. IWAA95, KEK-95-12, 1995.

[4] R.Pitthan, SLAC-PUB-7043, SLAC-PUB-8286, 1995, 99.

[5] R.Brinkmann, J.Rossbach, Nucl. Inst. Meth. Phys. Res. Sect. A 350, 8 (1994).

[6] S.Takeda, et al., KEK Prepr. 99-135, in Proc. IWAA 1999.

[7] R.Assmann, et al. SLAC-PUB-7303, 1996.

[8] A.Seryi, SLAC-PUB-8597, 2000.

[9] L.Hendrickson, et al., in these Proceedings.

[10] A.A.Michelson, Astrophysical Journal, volume XXIX, March 1914, Number 2

[11] NLC ZDR Design Group, SLAC Report-474 (1996).

[12] The NLC Collaboration, 2001 Report on the Next Linear Collider, SLAC-R-571, June 2001. 